\title{
Issues impairing the success of neural implant technology
}

doi:10.1533/abbi.2006.0055

\author{
A. Spiers, K. Warwick, M. Gasson and V. Ruiz \\ Department of Cybernetics, University of Reading, Reading, RG6 6AY, United Kingdom
}

\begin{abstract}
By monitoring signals from the central nervous system, humans can be provided with a novel extra channel of communication that can, for example, be used for the voluntary control of peripheral devices. Meanwhile, stimulation of neural tissue can bring about sensation such as touch, can facilitate feedback from external, potentially remote devices and even opens up the possibility of new sensory input for the individual to experience. The concept of successfully harnessing and stimulating nervous system activity is though something that can only be achieved through an appropriate interface. However, interfacing the nervous system by means of implant technology carries with it many problems and dangers. Further, results achieved may not be as expected or as they at first appear. This paper describes a comparative study investigating different implant types and procedures. It is aimed at highlighting potential problem areas and is intended to provide a useful reference explaining important tolerances and limits.
\end{abstract}

Key words: Implant technology, nerves, neural stimulation.

\section{INTRODUCTION: MOTIVATION FOR IMPLANTABLE NEUROPROSTHESIS}

The production of a reliable and efficient interface linking the human brain and/or nervous system with a computer network is now, given present-day technology and understanding, a realistic goal. Such a device opens up enormous potential opportunities both for therapeutic purposes and for, what might be described as, enhancements. However, this is a relatively new field of investigation, and hence not only do a variety of different implant types exist but also questions immediately arise as to their positioning, tolerances and effectiveness. Clearly, along with appropriate experimental trials, a basic selection of standard techniques and results needs to be established. Researchers looking into the same work, producing the same results and (occasionally) making the same mistakes needs to be avoided wherever possible. This article is an attempt to provide a starting point guide to implantation. It is hoped that further trials, results and subsequent experience will

Corresponding Author:

K. Warwick

Department of Cybernetics

University of Reading

Whiteknights, Reading, Berks RG6 6AY, United Kingdom

Tel: +44-118-378-8210; Fax: +44-118-378-8220

Email: k.warwick@reading.ac.uk then contribute to a continued growth of knowledge in the field.

For any individual with a condition such as Lou Gehrig's disease, the world is an environment that may be observed and understood but most probably interacted with only via yes/no eye blinks interpreted by a carer. For a person to be cognitively intact but to experience communication with such a low bandwidth is undoubtedly frustrating. Questions regarding food and comfort must be answered relatively slowly in binary while the individual remains unable to communicate easily any of his or her higher level thoughts. By moving one step beyond eye blinks, this would permit the individual a greater degree of interaction with his or her environment. Even the operation of a simple on/off bell to call a carer or nurse would return a degree of control.

Research has already enabled, by means of peripheral nervous system (PNS)-implanted devices, the integration of recording electrodes that allow prosthetic limbs to be controlled via nerve signals (Warwick et al. 2003). This includes feedback to individuals such that they can experience grasping forces applied by an articulated hand and subsequently stimulating such sensory inputs as ultrasonic sensors (Gasson et al. 2005; Warwick et al. 2005). This is a significant step from more traditional prostheses, which can be so difficult to use that in some cases users prefer to do without them altogether. Other successful applications include alerting individuals with paralysis to signals that 
the body is trying to communicate to the brain, such as an indication that their bladder is full (Hoffer 1999).

Implanting stimulating electrodes into the peripheral nerves can affect the control of muscles in order to bridge injury and reclaim some control of paralysed limbs. By stimulating nerves rather than muscles, a much lower current is required, reducing the necessity for cumbersome battery packs and allowing for a response of much higher granularity.

\section{HARDWARE CONSIDERATIONS}

The human nervous system is considered robust because its neuroplastic nature allows functional adaptation, given interior and exterior influences (Pascual-Leone et al. 2005). However, from a physical perspective, it is a delicate and complex structure that can be irreparably damaged. Given this, the measurement and/or stimulation of nerve fibres and brain tissue require a level of precision that must be achieved without compromising safety considerations.

The design of a neuroprosthesis requires an effective and efficient method of interfacing whilst remaining within the constraints imposed by microengineering technologies and a sensible budget. Just how effective the interface then is based on many variable characteristics affected by both the choice of manufacturing technique and the proposed application. Some of the key aspects are presented below.

\section{Site of implantation}

The purpose of an implantable device, such as a neuroprosthesis, is to either measure from or to stimulate the nervous system. Some implants are designed specifically for use in either the peripheral or central nervous systems (PNS or CNS); however, most available devices can be used with either of the two systems. A brief overview of the different implantable areas is provided below in order to identify particular features.

\section{Central nervous system: Motor cortex}

The brain can be considered to be divided loosely into regions that relate to functional and processing abilities. If one is required to derive a consciously controllable signal from the brain, it is necessary to target an area for which neuronal activity can be reliably and quantifiably evaluated. The motor cortex, which deals with movement, is ideal for this purpose, much more so than other areas of the brain used primarily for such functions as perception or memory.

Every time a (motor) movement is executed by an individual, an increase in neuronal firing occurs in a specified region of the motor cortex. These regions, which consist of clusters of neuronal colonies, are spatially mapped directly to areas of the body. It is therefore possible to target areas such as the arm or hand by obtaining measurements from the relevant region of the cortex. Numerous non-human implant studies have in fact already targeted the motor cortex with very promising results (Nicolelis et al. 2003; Chapin 2004).

There are several methods that can be used to measure brain activity. Those based on recording electrical activity generally record compound signals that originate from multiple sources. The compound activity recorded from a small population is referred to as a local field potential, although a grosser recording can be made non-invasively by electroencephalogram electrodes placed on the scalp. This surface method of detection however results in recordings that are difficult to localise and with slow response times for detecting voluntary activity (Donoghue 2002). A potentially more effective region for measurement of motorrelated activity is invasively within the 1.5 - to $2-\mathrm{mm}$ deep layer of grey matter close to the skull where neurons are densely populated.

Neural signals originate in the central (sub-thalamic) area of the brain and propagate outwards towards the skull. As they disperse into the relevant cortices, they become more task specific. Neuronal activity within the cortex can be observed to occur in progressing waves and in several locations at once via the granular/stellate neuronal cell interconnections that exist between neuronal colonies. Each colony may be roughly modelled as a vertical cylinder of approximately $1-\mathrm{mm}^{2}$ diameter.

\section{Peripheral nervous system}

The PNS encompasses the many nerves fibres that permit the CNS to communicate with sensory receptors (via afferent fibres) and motor effectors (via efferent fibres) located throughout the body. The major nerve trunks of the body (the median, sacral, etc.) consist of numerous nerve fascicles, which, in turn, are composed of nerve fibres (the axons of individual cells). A single nerve fascicle may contain many tens of thousands of such fibres. Typically, diameters of individual nerve fibres are between 2 and $20 \mu \mathrm{m}$. Because nerve fibres constitute the axon of a neuron, it is this region of the nerve trunk that is of specific interest when recording activity from the PNS. The epineurium, perineurium and endoneurium tissue layers that separate and insulate nerves and axons from one another, whilst providing excellent protective mechanisms, tend to hinder measurement of cellular activity.

Nerves in the PNS respond with, all or nothing, binary action potentials, although the pattern of activity for even simple movements is extremely complex.

\section{FEATURES OF A NEUROPROSTHESIS}

The key role of any neuroprosthesis is to provide a functional interface with the nervous system. For a neuroprosthesis to serve a useful purpose in rehabilitation engineering, it must be designed to remain in the body for an extended period of time (chronic implantation). This places further stringent criteria on its design and operational requirements. A prosthesis can be defined 


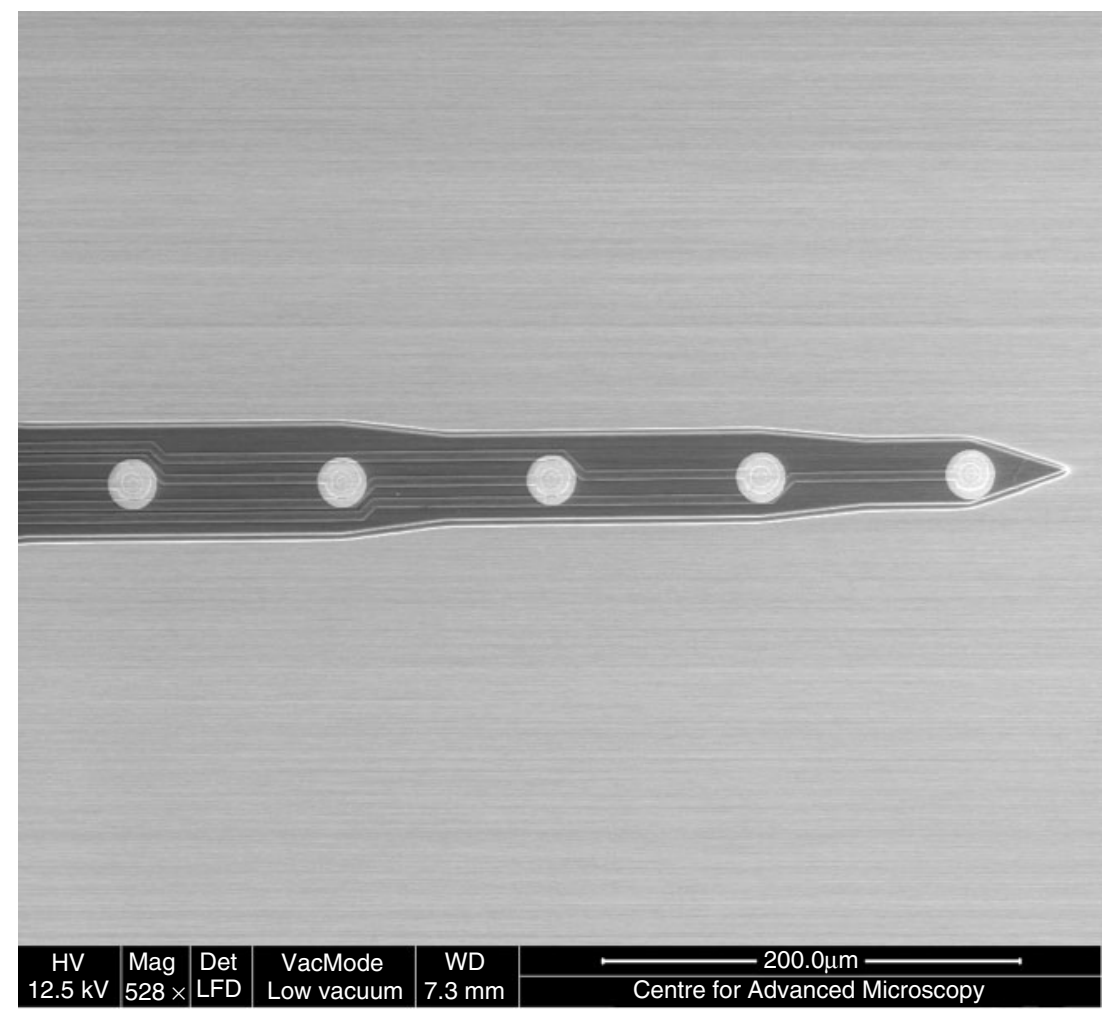

Figure 1 The end of a two-dimensional silicon NeuroNexus shaft probe with five iridium electrode sites.

as "an artificial body part" and as such it should be expected to exist in harmony with the rest of the body. Acute electrodes may well be designed primarily for temporary use in anaesthetised animals. Their requirements are significantly different from those of chronic electrodes, which must address the many concerns associated with long-term body reactions and the mechanical stress associated with mobile environments, particularly when human subjects are involved. These concerns and the features designed to approach them are now described.

\section{Accurate targeting}

It is quite possible to obtain some measure of brain activity using non-invasive scalp-mounted electroencephalogram electrodes, however, the usefulness of the signals actually measured is limited. In a similar way, extra-neural electrode devices exist (Jensen et al. 2001) for measurement of the compound action potential of groups of nerves that make up a nerve trunk. Although both devices have been used to provide quantifiable results, the generalised nature of data collection means that their resolution is low and repeatability of activity is difficult to achieve.

More accurate and higher resolution recording techniques could permit more 'degrees of freedom' for use in prostheses control, facilitating a higher level of interaction and thereby an improved quality of communication. The regions of the brain that may allow for this are the pyramidal and ganglionic layers. However, these layers are only $300 \mu \mathrm{m}$ in depth, and so present a challenge from an elec- trode insertion point of view. The practical likelihood with implantation is that an implanted electrode either will not reach the correct layer or will pass through it.

For acute measurement from the human brain, as is the case in deep-brain stimulation surgery, the patient is generally held fixed in a place whilst electrodes are carefully lowered into the appropriate regions of the brain by means of precise tools. Although this technique can deliver excellent implant positioning results, it is very time consuming and requires considerable quantities of specialised and expensive equipment. On top of this, the much extended operation time greatly increases the possibility of further problems for the recipient, especially in terms of infection.

Problems of targeting specific areas also arise in the PNS where the numerous insulating layers of tissue need to be bypassed. The type of electrode(s) employed can make a significant difference in success versus failure rates with regard to establishing a reasonable contact with a desired area or cell type. For example, employing a shaft electrode improves the likelihood of interfacing with the desired region of a nerve trunk or cortical area. With this type of electrode, multiple bi-directional electrode sites are concatenated on to a single needle-like shaft, making it possible to target several layers of tissue at once. In a typical shaft device (Figure 1), the 16 electrodes are spaced approximately $60-\mu \mathrm{m}$ apart, resulting in an overall measuring area spanning approximately $1.6 \mathrm{~mm}$. This implies that an electrode of this sort placed in the motor cortex may result in up to five electrodes in a relevant $300-\mu \mathrm{m}$ deep layer, which almost ensures that some of the electrodes will 


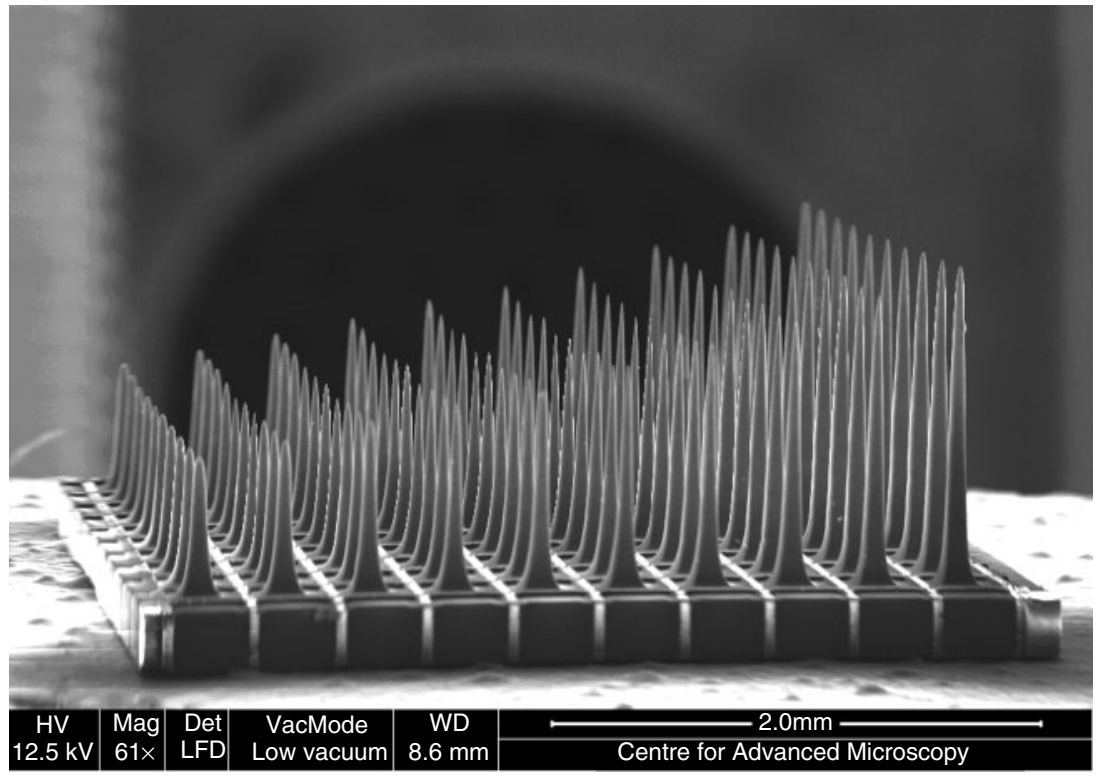

Figure 2 A Utah Slant Array with 100 electrode shafts of differing lengths for varying penetration depth.

reside proximate to either the ganglionic or pyramidal layers. This same approach also applies when implanting this type of multi-electrodes into the nerve trunks of the PNS. While a quantity of electrodes will undoubtedly reside in insulating tissue, there is a high likelihood that some of the sites will make contact with the nerve fibres themselves. Such an approach has the added benefits of reducing operation time and the need for accurate placement.

\section{Multiple recording sites}

Increasing the number of recording sites over the area of a single implant potentially allows for the collection of signals from neighbouring neurons and nerve fibres. As signals in the brain are in general not determined by individual neuronal activity, it is often necessary to observe the inhibition of neighbouring neurons and neuronal colonies (see the 'Site of implantation' section). It is reasonable to indicate that there are numerous methods of arranging electrodes points in terms of a single implant device. Shaft electrodes (Fig. 1) have the characteristic that they identify the vertical propagation of signals. Array devices mean while give access to lateral neuronal interaction, permitting the pinpointing of neural activity and observation of several colonies. A negative of this, though, is that it results in more difficult vertical positioning of the recording tip. Investigations are presently being carried out in the fabrication of an array of shaft electrodes (WIMS 2003) (permitting lateral inhabitation measurement at different cortical layers). It is though not yet possible to purchase them commercially, and hence actual test results and characteristic response information are not available.

The history of multi-site electrode arrays can be traced back to the practice of manually placing numerous electrodes into a particular location in the brain. Microfabrication techniques however now allow for the distances between active sites to be significantly smaller and uniform. The arrays are typically packaged into one device, which eases actual implantation and reduces the related operation time.

The way the nerve fibres are arranged in the PNS means that measurement from electrodes all implanted to the same fixed depth in a linear function could (in theory) simply result in a repetition of results, with slight time delays resulting in redundancy (Branner et al. 2001). To measure from multiple nerves using a multi-site electrode, it may therefore be preferred to arrange electrodes at varying depths. This is possible either with a shaft electrode or with a slanted electrode array (Fig. 2) with different shaftlength electrodes. It is worth adding a practical note here though that this problem of signal redundancy assumes an almost ideal implantation for a uniform shaft-length multi-electrode array. In practice, such an implantation is unlikely.

\section{Stability}

The adhesion of a chronic neuroprosthesis to its implant site and possible migration is a cause of considerable concern. Even movements of sub-300 $\mu \mathrm{m}$ will relocate an electrode to a different layer of the motor cortex. Given that the distance between synapses is only $20 \mu \mathrm{m}$, it takes an even smaller deflection $(\sim 0.1 \mu \mathrm{m})$ to divert measurement away from a particular neuron. "Micro-motion" of this sort can occur naturally, for example, the heartbeat-driven pulsing of the brain or simply everyday movement. Statically tethering a device to the skull (by putty or fixed frame) was one method of maintaining the position of an implant. However, the pulsing motion of the brain not only causes repeated separation of electrodes and measurement site but also leads to lacerations, as mobile soft brain tissue tears against the rigid device. Alternative 'floating' electrode 


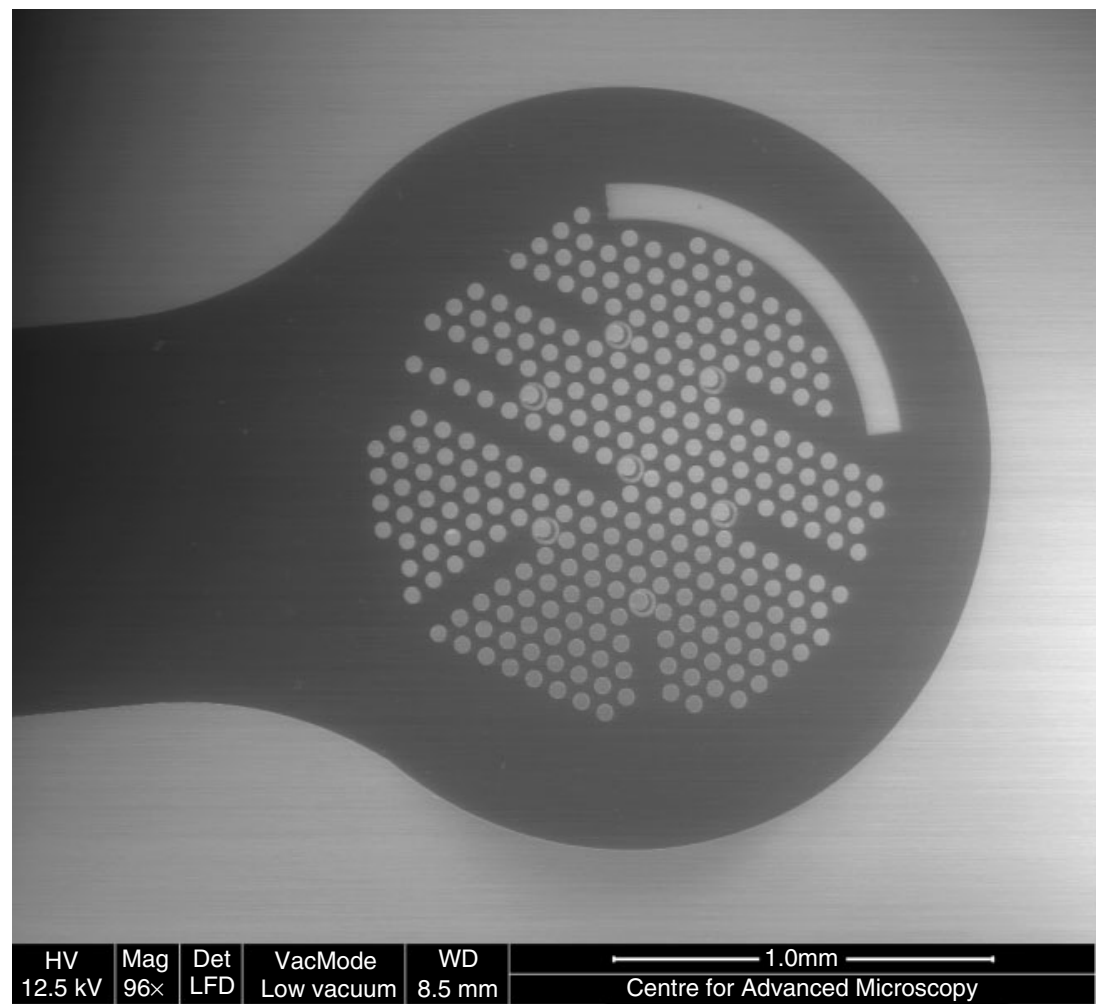

Figure 3 A polymer sieve electrode containing a series of perforations that allow small populations of nerve fibres to regenerate through the device. Seven of these sites, which display a light ring around the hole circumference, are active electrodes.

devices substitute the long rigid shaft of acute designs with a flexible tether connected to a small electrode-containing structure. This development permits the interface to move freely with the brain, adhering to measurement sites and reducing trauma by absorption rather than opposition of motion.

Polymer devices, which are available in many different forms such as shaft electrodes (Stieglitz et al. 2001), use their inherent flexibility to fit the contours and absorb the brain motion. Penetrating tissue with a flexible device does though lead to the problem of buckling. To overcome this, materials research is therefore ongoing.

Methods of temporary stiffening do exist (Kipke 2002). By coating with a specific weight of polyethylene glycol, one is able to specify a temperature (i.e. $45^{\circ} \mathrm{C}$ : human body; $15^{\circ} \mathrm{C}$ : amphibian) at which the coating dissolves to leave a flexible device. Dissolved polyethylene glycol fluid is harmlessly broken down and absorbed.

Holes and cavities are sometimes machined into implants with the intention of anchoring implants via biological symbiosis. In the case of sieve electrodes (Fig. 3), an interface is made by a peripheral nerve transection, which then regenerates through the many small holes that constitute the sieve (some of which act as electrodes). Although these devices are highly stable (providing they are not subject to forceful disturbance), they are susceptible to inexact targeting due to indeterminate nature of regeneration. It is quite possible that when looking to stimulate motor efferent fibres, most electrode connections may have unfortunately been made with afferent (sensory reception) fibres.

\section{Tissue trauma}

Neuroprosthesis deals with some of the most delicate areas of the body. It is therefore important that tissue damage is kept to an absolute minimum, both during and after implantation. It is normally the case that implantation of 'traditional' needle-like microneurographic electrodes simply involves pushing devices into tissue and removing them once the acute measurements have been taken. If a device is suitably stiff and thin, it may even be possible to enter into the brain via the pia and dura mater. Where chronic insertion is concerned, the motion of stiff electrodes can have a cutting effect that destroys tissue. If a stiff implant of this sort is statically fixed to the skull of the patient, then the pulsing effect of the brain against the stiff implant (see section 2.2.3) will simply cause the device to bore out the area of tissue originally intended for interfacing.

Flexible devices adhere better to an implant site; however, they suffer from a number of penetration difficulties, such as bending and even potential breakages. Very similar problems in fact occur for large surface area devices. As a means of getting round this, in the case of the electrode array shown in Figure 2, it is necessary to use a highvelocity insertion device (an impactor) to minimise bruising of tissue. 
It is important that devices cut into as little tissue as possible in the brain or PNS. These lacerations are not only damaging but also the subsequent formation of scar tissue makes adhesion difficult and micromotion a greater concern. Many devices possess rounded edges and tips, designed to separate cells on implantation rather than destroying them. That said, the damage that results from the insertion of the electrode into the tissue is negligible compared with the electrochemical electrode effects that can occur (Fung et al. 1998). Indeed, the electrode tip shape is generally shown to have little effect on tissue damage, although it is highly relevant with respect to current distribution during stimulation (Gimsa et al. 2005). Biphasic stimulation is generally used to balance the effects of anodic and cathodic electrode reactions, and, indeed, less tissue damage is typically found when using biphasic as opposed to single-phase stimulation (Fung et al. 1998).

While damage to the PNS is undesirable, it is often less damaging long-term compared with trauma in the CNS. This is in part due to the regenerative nature of PNS nerves. Although nerves in the brain are also able to regenerate, the large quantity of inhibitors in the CNS prevents such actions.

\section{BIOCOMPATIBILITY}

If the relationship between a neuroprosthesis and its organic environment is incompatible, this will likely lead to poor results or even a harmful biological reaction. Poor biocompatibility can lead to tissue inflammation. In the case of brain interfaces, this is a serious concern, as the swelling of the brain within its tight cranial chamber could lead to the compression of cortical tissue against the skull and very significant tissue damage. In other circumstances, it may be the implant that suffers as a result.

\section{Hostile cerebral environment}

The highly corrosive environment of the human body can be damaging to electrodes of particular material types. Electrode corrosion, deterioration and the production of neurotoxic compounds may particularly be expected when electrodes are used for stimulation. One of the more noticeable characteristics of electrode degradation is a significant change in device impedance, that is, the interface impedance increases dramatically. Much of the current research aims to measure the effects following exposure to such corrosive conditions, both with and without stimulation (Gimsa et al. 2005), such that the relative susceptibility to long-term degradation, which is clearly detrimental to chronic implant suitability, can be determined.

\section{Gassing}

Another major concern is the generation of chlorine, reactive oxygen or hydrogen during stimulation via the process known as gassing. These chemicals lead to toxification, acidification or highly reactive hydrogenated compounds, respectively. Gassing will inevitably damage or kill tissue surrounding the electrode site. Effects can be reduced by use of lower currents, low-pulse frequencies and nonpolarizing electrodes with larger surface areas (to reduce local current flow). Note however that reducing current will reduce stimulation effectiveness, while larger surface areas will lead to loss of neuronal pinpointing.

\section{Encapsulation}

The human body defends itself from potentially hazardous foreign objects via a process known as encapsulation, where a layer of neuroglia cells is formed over the object via a process known as gliosis. This is similar to the attachment of white blood cells to a flesh wound. By its very nature, an invasive electrode is a foreign body, and its relative biocompatibility will dictate the level of host reaction to it. However, by being encapsulated within a neuroglial capsule, the impedance of a device is increased significantly, and thus the recording ability of the electrode suffers (Turner $e t a l$. 1999). A benefit of capsule formation however is improved implant stability. In recent years, researchers of brain interfaces have looked to actually harness the beneficial stability properties of encapsulation while simultaneously preventing gliosis at the implant site. Use of bioactive coatings (Kipke 2002) and nerve tropics from the patient's own body (Kennedy et al. 2000) has been found to encourage neuron growth on to interface sites, giving a better contact than can simply be obtained by placing electrodes adjacent to the targeted area. In cases where neurons are not grown on to interface sites, it is important that there is a good electrochemical relation between an electrode and the cerebral fluid.

\section{Protein poisoning}

When a current is passed through an electrode, inherently 'sticky' proteins are attracted to the electrode. The resulting build up of proteins eventually covers the electrode, significantly degrading signal quality. In some stimulating procedures, the build up of proteins is not a problem, as the relatively large stimulation current regularly destroys these molecules. It is possible therefore to 'clean' a poisoned measurement electrode by a brief passage of stimulation current. However, more research in this area is necessary to determine the susceptibility of various implants to protein poisoning while also establishing the time required before cleaning must take place (estimated to be several days). A concern is that the current necessary for an effective cleaning may also damage the nerve tissue.

\section{Thermodynamic properties}

It is characteristic of materials to undergo an increase in temperature due to current flow. In the brain, a temperature increase of even $1^{\circ} \mathrm{C}$ can cause tissue damage. It is therefore highly important to establish that using a neu- 
roprosthesis for stimulation purposes will not cause tissue damage via any thermodynamic response.

\section{Electrical characteristics}

In the environment of the CNS, it is key that the electrical properties of any measuring device permit the optimum acquisition and transferral of activity with minimal degradation and loss of signals. It is also the case with stimulation that it is important to monitor the precise amounts of currents applied to the nervous system while also maintaining confidence regarding the consistency of delivered waveform. Many electrical properties of implanted electrodes are yet to be fully investigated in order to evaluate electrode performance, particularly how they vary over time. Some of the key aspects are presented below.

\section{Impedance}

Impedance plays a major role in both stimulation and measurement. The impedance of a device will most likely vary during its lifetime, especially if it is being used for stimulation. When an implant becomes encapsulated by biofilm, its impedance increases and broader recordings are taken from a more generalised area. This reduced precision is thought to be due to distribution of the signal through the capsule (also accounting for signal degradation).

\section{Signal distortion}

Passage of a signal through non-linear circuitry can result in the acquisition of unwanted distortion. With neural prosthesis already subject to large amounts of noise (due to small initial signals and adjacent activity within the nervous system), it is essential that such sources are kept to a minimum. Harmonics of a system may be determined by signal processing such as fast Fourier transforms. Gaining knowledge of each implant's properties should make it possible to use signal-processing methods to deconstruct and remove unwanted distortion, thus giving access to the original nerve signals.

\section{DC current leakage}

DC current leakage can cause physical 'shocking' sensations when applied to the nervous system. This effect has been observed from fractured deep-brain stimulation electrodes (Young 1997). In this case, the electrode ceased to serve its chronic pain suppressing function and served only to agitate the patient. Inevitably, DC current leakage will damage tissue, and is thus unacceptable for a medical device.

\section{Charge injection}

A result of stray capacitance, charge injection occurs when an anomalous quantity of charge is injected from mismatched charges over transformer elements of a circuit. The effect of this would be an increase in measured charge that did not stem from patient activity or was not desired for stimulation purposes. It is reminded that the nervous system is highly precise and delicate and that damage from overstimulation will destroy cells.

\section{Hardware signal limitations}

Signals produced by the nervous system are of a very low magnitude and time period. As such, detection is inevitably very difficult, due not only to signal processing considerations but also to the susceptibility to disturbances. Concerns of noise can often be reduced by amplifying a signal close to its origin, thus reducing vulnerability of a signal as it propagates along a wire and into a signal-processing unit. Prior to the amplification stage, there is a risk that signals may be absorbed by elements of parasitic capacitance that reside within an implant. Absorption of a signal in this way will result in severely reduced neuroprosthetics functionality.

Determining areas of signal loss prior to implantation allows one to identify areas of concern in the implant that may otherwise not produce signals mysteriously or failed to elicit results from stimulation. It is therefore essential that useable frequencies (those that disregard absorbing elements) are determined prior to implantation. Performing frequency sweeps on implants immersed in saline permits identification of present and absent signals to show useable frequencies.

It is also necessary to determine maximum stimulation parameters for any given electrode type. As electrodes are designed for small signals only, large current spikes may destroy conducting electrode tips/pads. Because of the difficulties and trauma associated with implantation, it is better if this current is determined and avoided prior to implantation rather than having to face replacement of a faulty device.

\section{CONCLUSIONS}

Interfacing with the nervous system by means of implant technology is a practice that carries a low margin of error, both in terms of patient safety and in obtaining useful and meaningful results. As an effective means to reduce the risks of inefficient or even dangerous implantation, it is necessary to obtain a high degree of confidence in the hardware employed for the actual biological-technological interface. As an example, failure to identify such features as parasitic capacitance prior to implantation can lead to confusion and wasted time, as researchers attempt to determine why certain stimulation frequencies are not apparently achieving their expected effects.

By a comparative study of hardware data, researchers are subsequently able to select implants with a greater confidence and to design their experiments by taking into account consideration of the limitations and thresholds of the different devices being investigated. This article has highlighted some of the key areas that need to be considered by researchers in the field. Armed with such a reference, it is hoped that future work will be better informed with 
regard to initial hardware testing, and hence researchers will be able to divert their resources more directly towards post-implantation testing.

\section{ACKNOWLEDGMENTS}

This work has been funded in part by the Geneva Institut International de Recherche en Paraplégie (IRP).

\section{REFERENCES}

Branner A, Stein RB, Norman RA. 2001. Selective stimulation of cat sciatic nerve using an array of varying-length microelectrodes. 7 Neurophysiol, 85:1585-94.

Chapin JK. 2004. Using multi-neuron population recordings for neural prosthetics. Nat Neurosci, 7:452-4.

Donoghue JP. 2002. Connecting cortex to machines: Recent advances in brain interfaces. Nat Neurosci Suppl, 5:1085-8.

Fung SH, Burstein D, Born RT. 1998. In vivo microelectrode track reconstruction using magnetic resonance imaging. 7 Neuros $i$ Methods, 80:215-24.

Gasson M, Hutt B, Goodhew I, et al. 2005. Invasive neural prosthesis for neural signal detection and nerve stimulation. Int $\mathcal{F}$ Adaptive Control Signal Process, 19(5):365-75.

Gimsa J, Habel B, Schreiber U, et al. 2005. Choosing electrodes for deep brain stimulation experiments-electrochemical considerations. 7 Neurosci Methods, 142:251-65.

Hoffer A. 1999. New technology will help control paralysed limbs. Simon Fraser University Nems, 14(1). Accessed 7 January 1999. URL: http://www.sfu.ca/mediapr/sfnews/1999/Jan7/ hoffer.html

Jensen W, Lawrence SM, Riso RR, et al. 2001. Effect of initial joint position on nerve cuff recordings of muscle afferents in rabbits. IEEE Trans Neural Syst Rehabil Eng, 9(3):256-73.
Kennedy P, Bakay R, Moore M, et al. 2000. Direct control of a computer from the human central nervous system. IEEE Trans Rehabil Eng, 8(2):198-202.

Kipke DR. 2002. Advanced neural implants and control. Accessed June 2005. URL: http://www.darpa.mil/dso/thrust/biosci/ bim/briefings/ASU_Kipke.PDF

Nicolelis MAL, Dimitrov D, Carmena JM, et al. 2003. Chronic, multisite multielectrode recordings on macaque monkeys. PNAS, 100(19):11041-6.

Pascual-Leone A, Amedi A, Fregni F, et al. 2005. The plastic human brain cortex. Annu Rev Neurosci, 28:377-401.

Stieglitz T, Heiduschka P, Schuettler M, and Gross M. 2001. Reducing insertion sites of penetrating multipolar shaft electrodes by double side electrode arrangement. In Proc 23rd IEEE International Conference of the Engineering in Medicine and Biology Society, 25-28 October, Istanbul, Turkey, Vol. 4, pp. 3426-29.

Turner JA, Shain W, Szarowski DH, et al. 1999. Cerebral astrocyte response to micromachined silicon implants. Exp Neurol, 156:33-49.

Warwick K, Gasson M, Hutt B, et al. 2003. The application of implant technology for cybernetic systems. Arch Neurol, 60(10):1369-73.

Warwick K, Gasson M, Hutt B, et al. 2005. An attempt to extend human sensory capabilities by means of implant technology. In Proc IEEE International Conference on Systems, Man and Cybernetics, Hawaii, 10-12 October, 2005, p. $1663-8$.

WERC IMS Annual Report. 2003. Ann Arbor, MI: Center for Wireless Integrated Microsystems (WIMS), The University of Michigan. Accessed January 2003. URL: http://www. wimserc.org/about/annual_report.php

Young RF. 1997. Deep-brain stimulation for the treatment of chronic pain. Curr Sci, 1:182-91. 

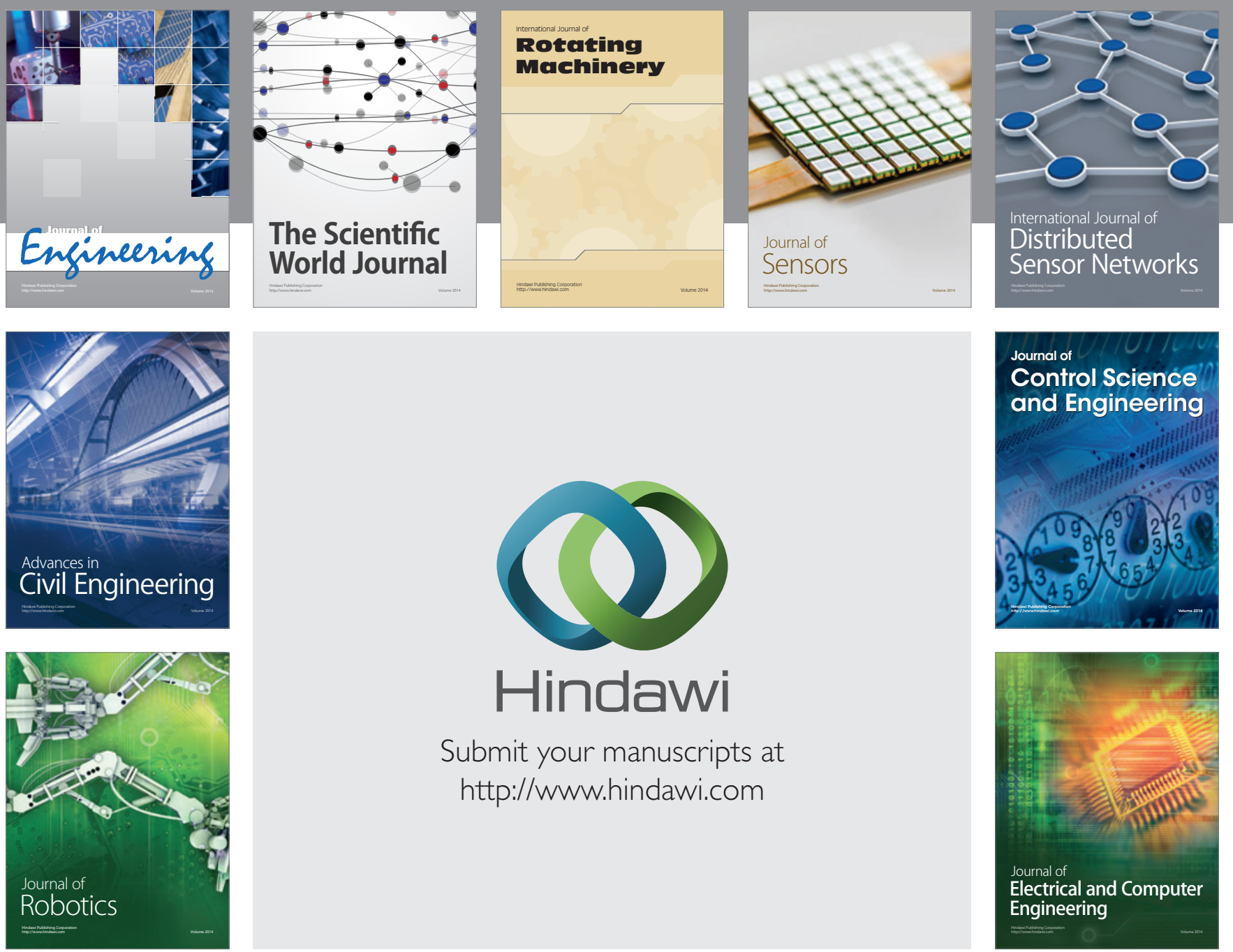

Submit your manuscripts at

http://www.hindawi.com
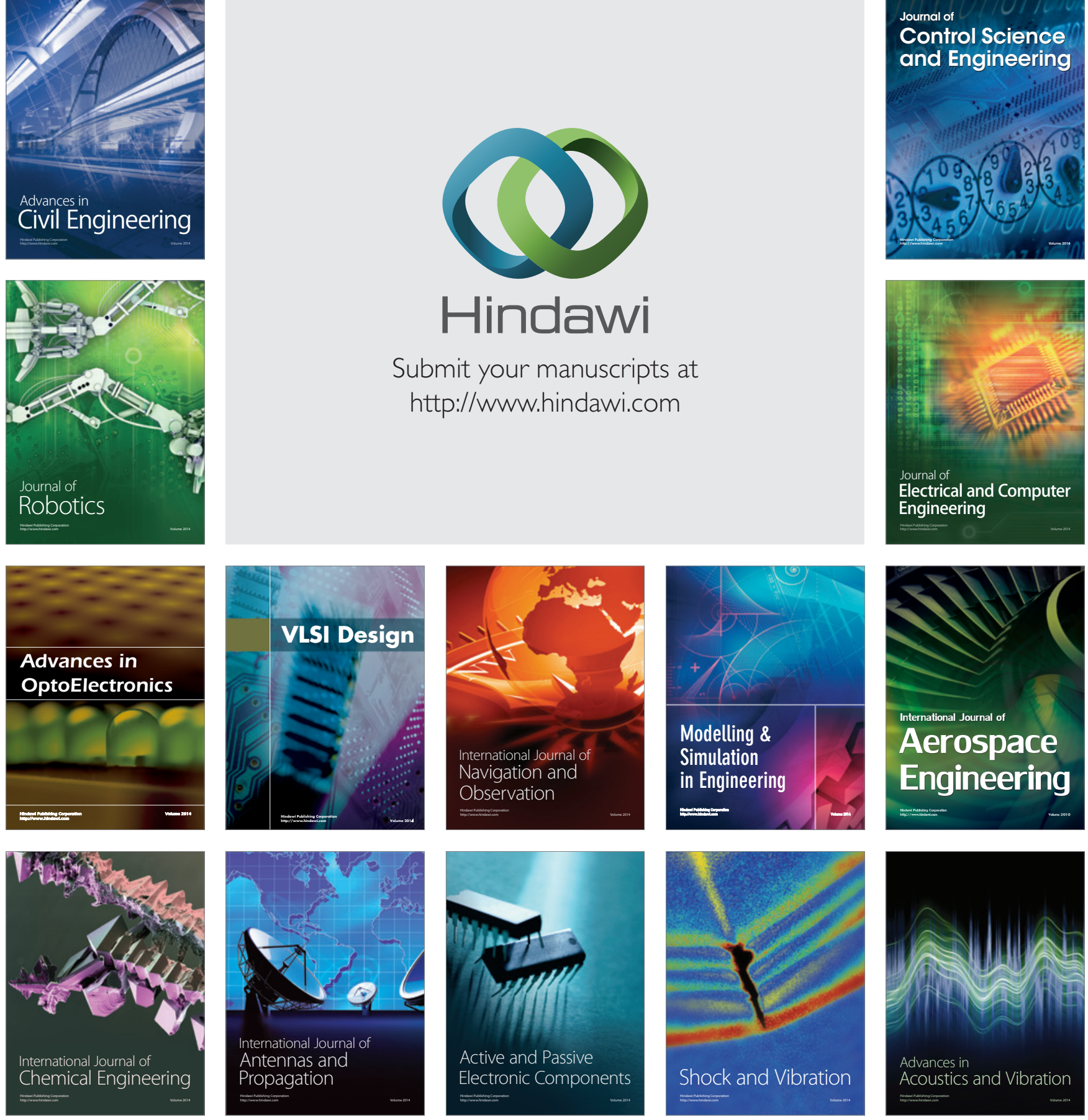